FERNANDA BORGES DIIIGOW ${ }^{1}$

Cristiane de Frettas Paganoti ${ }^{1}$

Rafaela Alkmin da Costa ${ }^{1}$

Rossana Pulcinel Vierira Francisco ${ }^{2}$

MARCELO ZUGAiB ${ }^{2}$

Artigo Original

\title{
Influência da amamentação nos resultados do teste oral de tolerância à glicose pós-parto de mulheres com diabetes mellitus gestacional
}

\author{
The influence of breastfeeding in postpartum oral glucose tolerance \\ test in women with recent gestational diabetes mellitus
}

\section{Palavras-chave \\ Amamentação \\ Diabetes mellitus gestacional \\ Puerpério \\ Diabetes mellitus tipo 2 \\ Fatores de risco \\ Keywords \\ Breastfeeding \\ Diabetes, gestational \\ Postpartum period \\ Diabetes mellitus, type 2 \\ Risk factors}

\section{Resumo}

OBJETIVO: Avaliar a influência da amamentação nos resultados do teste oral de tolerância à glicose pós-parto (TTGp) de mulheres que apresentaram diabetes gestacional atendidas em unidade terciária do município de São Paulo. MÉTODOS: Foram obtidos dados de pacientes com diabetes gestacional no período de janeiro a dezembro de 2014. As informaçães foram obtidas por meio de acesso aos prontuários eletrônicos e pelo contato telefônico. Seguindo os critérios de inclusão adotados, 132 pacientes foram elegíveis para o estudo. Para análise estatística dos dados, as pacientes foram divididas em dois grupos, segundo a informação de terem ou não amamentado. Foram utilizados os testes t de Student, de Mann-Whitney, do $\chi^{2}$ e exato de Fisher, dependendo do tipo de variável analisada. Foram considerados estatisticamente significativos testes com p<0,05. RESULTADOS: Das 132 pacientes incluídas no estudo, 114 amamentaram e 18 não amamentaram. Em ambos os grupos, houve um predomínio de pacientes na faixa do sobrepeso e/ ou obesidade. As pacientes que amamentaram apresentaram índice de massa corporal (IMC) pré-gestacional menor que as que não amamentaram ( $\mathrm{p}=0,006)$. No grupo que não amamentou, a idade gestacional de introdução de insulina foi mais precoce $(23.21 \pm 4.33$ versus $28.84 \pm 6$. 17; $(p=0,04)$ e o valor médio da glicemia de jejum

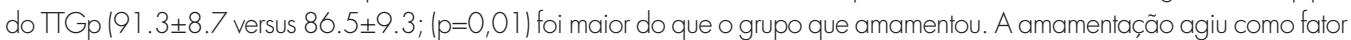
protetor para o desenvolvimento de intolerância à glicose no $T G p(O R=0,27 ; I C 95 \%$ 0,09-0,8). Pela regressão logística, a amamentação mostrou-se ser fator protetor independente. CONCLUSÃO: Houve relação estatisticamente significativa entre a amamentação e a diminuição do risco de desenvolver intolerância à glicose. Esse ato deve ser estimulado, visto que é uma intervenção efetiva de baixo custo e fácil acesso a todas as pacientes no puerpério.

\section{Abstract}

PURPOSE: To determine the influence of breastfeeding on the results of a postpartum oral glucose tolerance test in women recently diagnosed with gestational diabetes mellitus. METHODS: The data were obtained from the electronic medical records of the Endocrinopathy Sector during pregnancy, HCMED laboratory system of Hospital das Clínicas of São Paulo, and by telephone. According to the inclusion criteria adopted, 132 patients were eligible for the study. For statistical analysis, the patients were divided into two groups according to whether or not they breastfed. The results were analyzed by the Student t-test and by the Mann-Whitney, Chi-square and Fisher's exact tests, depending on the variable analyzed, with the level of significance set at $p<0.05$. RESULTS: Of the 132 patients included in the study, 114 breastfed and 18 did not. Most of the patients in both groups were overweight or obese. The breastfeeding group had a lower pre-pregnancy Body Mass Index than the non-breastfeeding group ( $p=0.006$ ). Insulin was introduced earlier in the group that did not breastfeed $(23.21 \pm 4.33$ versus $28.84 \pm 6.17 ; p=0.04)$. The group that did not breastfeed had a higher mean postpartum fasting glucose value in the oral glucose tolerance test than the group that breastfed $(91.3 \pm 8.7$ versus $86.5 \pm 9.3 ; p=0.01)$. Breastfeeding acted as a protective factor against the development of glucose intolerance in the postpartum oral glucose tolerance test $(O R=0.27 ; 95 \% \mathrm{Cl} 0.09-0.8)$. By logistic regression, breastfeeding was shown to be an independent protective factor. CONCLUSION: There was a statistically significant relationship between breastfeeding and a decreased risk of developing glucose intolerance. Breastfeeding should be encouraged because it is an effective, low cost intervention easily accessible to all patients during the postpartum period.

Correspondêncio

Fernanda Borges Diigow Instituto Central do Hospital das Clinicas Avenida Doutor Enéas de Carvalho Aquiar, 255, $10^{\circ}$ andar - Cerqueira César

(EP: 05403-000

São Paulo (SP), Brasil

Recebido

$25 / 08 / 2015$

Aceito com modificacōes

$24 / 09 / 2015$
Disciplina de Obstetrícia do Departamento de Obstetrícia e Ginecologia do Hospital das Clínicas da Faculdade de Medicina da Universidade de São Paulo - USP - São Paulo (SP), Brasil.

'Disciplina de Obstetrícia do Departamento de Obstetrícia e Ginecologia, Faculdade de Medicina, Universidade de São Paulo USP - São Paulo (SP), Brasil.

2Departamento de Obstetrícia e Ginecologia, Faculdade de Medicina, Universidade de São Paulo - USP - São Paulo (SP), Brasil.

Conflito de interesses: não há 


\section{Introdução}

O diabetes mellitus gestacional (DMG) é uma desordem metabólica multifatorial definida como qualquer grau de intolerância a carboidratos diagnosticado pela primeira vez na gestação, desde que não atenda aos critérios diagnósticos para diabetes mellitus (DM) utilizados fora do período gestacional ${ }^{1}$. Caracteriza-se por aumento da resistência periférica à insulina decorrente do aumento de hormônios placentários contrarreguladores da insulina, como o lactogênio placentário, o cortisol, o estrogênio, a progesterona e a prolactina ${ }^{1}$.

O estudo multicêntrico Hyperglycemia and Adverse Pregnancy Outcome (HAPO Study) ${ }^{2}$, envolvendo 25.505 gestantes em 15 centros e 9 países, correlacionou os níveis glicêmicos com desfechos perinatais desfavoráveis e, a partir de seus resultados, a International Association of the Diabetes and Pregnancy Study Groups (IADPSG) ${ }^{3}$ adotou novos valores para critérios diagnósticos do DMG, baseados nos valores do teste oral de tolerância à glicose (TTG) com $75 \mathrm{~g}$ de dextrose entre a $24^{\mathrm{a}}$ e a $28^{\mathrm{a}}$ semanas de gestação, a saber: glicemia de jejum (GJ) maior ou igual a $92 \mathrm{mg} / \mathrm{dL}$; $1 \mathrm{~h}$ após sobrecarga maior ou igual a $180 \mathrm{mg} / \mathrm{dL} ; 2 \mathrm{~h}$ após sobrecarga maior ou igual a $153 \mathrm{mg} / \mathrm{dL}$.

Dessa forma, apenas um valor alterado já seria suficiente para o diagnóstico de DMG. Com isso, a GJ, na primeira consulta de pré-natal, passou a ser obtida de todas as gestantes, sendo considerado DMG valores entre 92 e $125 \mathrm{mg} / \mathrm{dL}$. O TTG entre a $24^{\mathrm{a}}$ e a $28^{\mathrm{a}}$ semanas de gestação seria reservado para aquelas pacientes com GJ menor que $92 \mathrm{mg} / \mathrm{dL}$.

A frequência dessa intercorrência gestacional encontra-se em ascensão e, nos Estados Unidos, aproximadamente 7\% de todas as gestações coexistem com DMG, resultando em mais de 200.000 casos por ano ${ }^{4}$. No Brasil, a incidência é de 7,6\% em mulheres com mais de 20 anos atendidas no Sistema Único de Saúde (SUS) ${ }^{5}$. Devido à implementação de novos critérios diagnósticos pela IADPSG, houve um aumento no número de casos diagnosticados, podendo atingir de 15 a $20 \%$ das gestantes ${ }^{6}$.

Estudos demonstram que, no período puerperal, mulheres que tiveram DMG têm risco sete vezes maior de desenvolver diabetes mellitus do tipo 2 (DM2). Dessa forma, recomenda-se que todas as pacientes com diagnóstico de DMG sejam submetidas ao TTG entre 6 e 8 semanas após o parto, para rastreamento inicial de intolerância à glicose ou DM2 ${ }^{7}$. No nosso serviço, observou-se que no teste oral de tolerância à glicose pós-parto (TTGp) 25\% das pacientes com DMG desenvolveram intolerância à glicose, e 5\%, DM2, entre os anos de 2003 e $2009^{8}$.

Há evidências de que o aleitamento materno é um fator de proteção para o desenvolvimento de intolerância à glicose e DM2 em mulheres que tiveram $\mathrm{DMG}^{9-18}$.
Os mecanismos dessa proteção ainda não estão completamente estabelecidos. Estudos realizados em animais revelam que a prolactina aumenta a proliferação das células beta pancreáticas e, com isso, eleva a produção de insulina. Além disso, existem efeitos benéficos da lactação em curto prazo, como a diminuição da glicemia e, consequentemente, da insulinemia, devido ao uso da glicose para a produção láctea, bem como diminuição da lipotoxicidade pela mobilização de lipídios intracelulares dos hepatócitos e miócitos para a lactogênese ${ }^{16}$. A amamentação aumenta o gasto energético e contribui para a perda de peso e a diminuição da resistência periférica à insulina ${ }^{17}$.

Um estudo teve por objetivo avaliar a associação da frequência da amamentação com a glicemia, a insulinemia e a intolerância à glicose no TTG realizado de 6 a 9 semanas após parto em mulheres que tiveram $\mathrm{DMG}^{18}$. Ao final da avaliação, observou-se melhora dos níveis de GJ e níveis mais baixos de insulina. Isso demonstra que a lactação pode apresentar efeitos favoráveis sobre o metabolismo glicêmico e sensibilidade à insulina, o que, por sua vez, pode reduzir o risco de diabetes após gestações complicadas por DMG.

Considerando os benefícios da amamentação, sobretudo no que diz respeito ao seu possível efeito protetor no desenvolvimento de DM2, o presente estudo teve por objetivo avaliar a influência da amamentação nos resultados do TTGp de mulheres que apresentaram DMG.

\section{Métodos}

Trata-se de um estudo de coorte retrospectiva no qual foram avaliados os dados das pacientes com DMG acompanhadas durante o pré-natal no Setor de Endocrinopatias e Gestação da Clínica Obstétrica do Hospital das Clínicas da Faculdade de Medicina da Universidade de São Paulo (HC-FMUSP), no período de janeiro a dezembro de 2014. Por se tratar de uma análise retrospectiva de banco de dados, não caracterizando nenhum risco aos sujeitos da pesquisa, não foi aplicado o termo de consentimento livre e esclarecido.

Os dados utilizados foram obtidos por meio de acesso ao prontuário eletrônico do Setor de Endocrinopatias na gestação e por contato telefônico. Os dados laboratoriais foram obtidos por meio de acesso ao sistema de laboratório HCMED do HC-FMUSP.

As pacientes foram orientadas a realizar o TTG por volta de 40 dias após o parto e retornaram ao ambulatório para revisão do resultado e para orientações de puerpério. Nesse momento foram obtidas informações a respeito da amamentação (se aleitamento materno exclusivo, misto ou não amamenta). O aleitamento materno exclusivo é definido se apenas o leite materno é oferecido para alimentar 
o recém-nascido, e o misto quando são oferecidos leite materno e fórmulas lácteas.

Os critérios de inclusão do estudo foram: gestação única; diagnóstico de DMG de acordo com critérios diagnósticos propostos pela IADPSG ${ }^{3}[\mathrm{GJ}$ inicial $(\geq 92 \mathrm{mg} / \mathrm{dL}$ ) ou TTG entre 24 e 28 semanas de gestação (GJ $\geq 92 \mathrm{mg} / \mathrm{dL}$ e/ou $1 \mathrm{~h}$ pós-sobrecarga $\geq 180 \mathrm{mg} / \mathrm{dL}$ e/ou $2 \mathrm{~h}$ pós-sobrecarga $\geq 153 \mathrm{mg} / \mathrm{dL}$ )]; obtenção das informações referentes à amamentação no pós-parto (amamentação: sim ou não); obtenção dos resultados TTGp. Foram excluídas do estudo pacientes com cirurgia bariátrica prévia à gestação, com dados insuficientes no prontuário eletrônico do Sistema de Endocrinopatias, com intolerância à glicose previamente à gestação e aquelas em que não foi possível obter informações sobre a amamentação e sobre os resultados do TTGp. Entretanto, as pacientes excluídas devido à falta de dados laboratoriais apresentavam características clínicas e epidemiológicas semelhantes às que foram incluídas no presente estudo.

Os resultados do TTGp foram assim considerados: normal (GJ $\leq 99 \mathrm{mg} / \mathrm{dL}$ e $2 \mathrm{~h}$ após sobrecarga $\leq 139 \mathrm{mg} / \mathrm{dL}$ ) ou alterado ([GJ 100-125 mg/dL ou 2 h após sobrecarga $140-299 \mathrm{mg} / \mathrm{dL}$ — intolerância à glicose $] /[\mathrm{GJ} \geq 126 \mathrm{mg} / \mathrm{dL}$ ou $2 \mathrm{~h}$ após sobrecarga $\geq 200 \mathrm{mg} / \mathrm{dL}$ - DM2]).

Para análise estatística dos dados, as pacientes foram dividias em dois grupos, segundo a informação de terem ou não amamentado: amamentação sim (AS) e amamentação não (AN). As variáveis foram analisadas e comparadas segundo essa divisão. Para variáveis categóricas, foram utilizadas medidas de número e de frequência, enquanto as variáveis contínuas foram descritas em termos de média, mediana, desvio padrão (DP). As variáveis quantitativas foram inicialmente testadas quanto à sua distribuição em normal e não normal, por meio do teste de KolmogorovSmirnov. Aquelas com distribuição normal foram comparadas utilizando-se o teste de $t$ de Student, enquanto as de distribuição não normal foram comparadas utilizando-se o teste de Mann-Whitney. As variáveis qualitativas foram comparadas utilizando-se teste do $\chi^{2}$ ou teste exato de Fisher. Foi realizado o teste de regressão logística para avaliar quais variáveis estavam independentemente relacionadas ao desfecho. Foram considerados estatisticamente significativos os testes com $\mathrm{p}<0,05$.

\section{Resultados}

A busca inicial no Sistema de Endocrinopatias e Gestação identificou 272 pacientes que tiveram o diagnóstico de DMG no ano de 2014. Conforme o fluxograma (Figura 1), foram excluídas 140 pacientes (3 com intolerância à glicose previamente à gestação, 2 com antecedente de

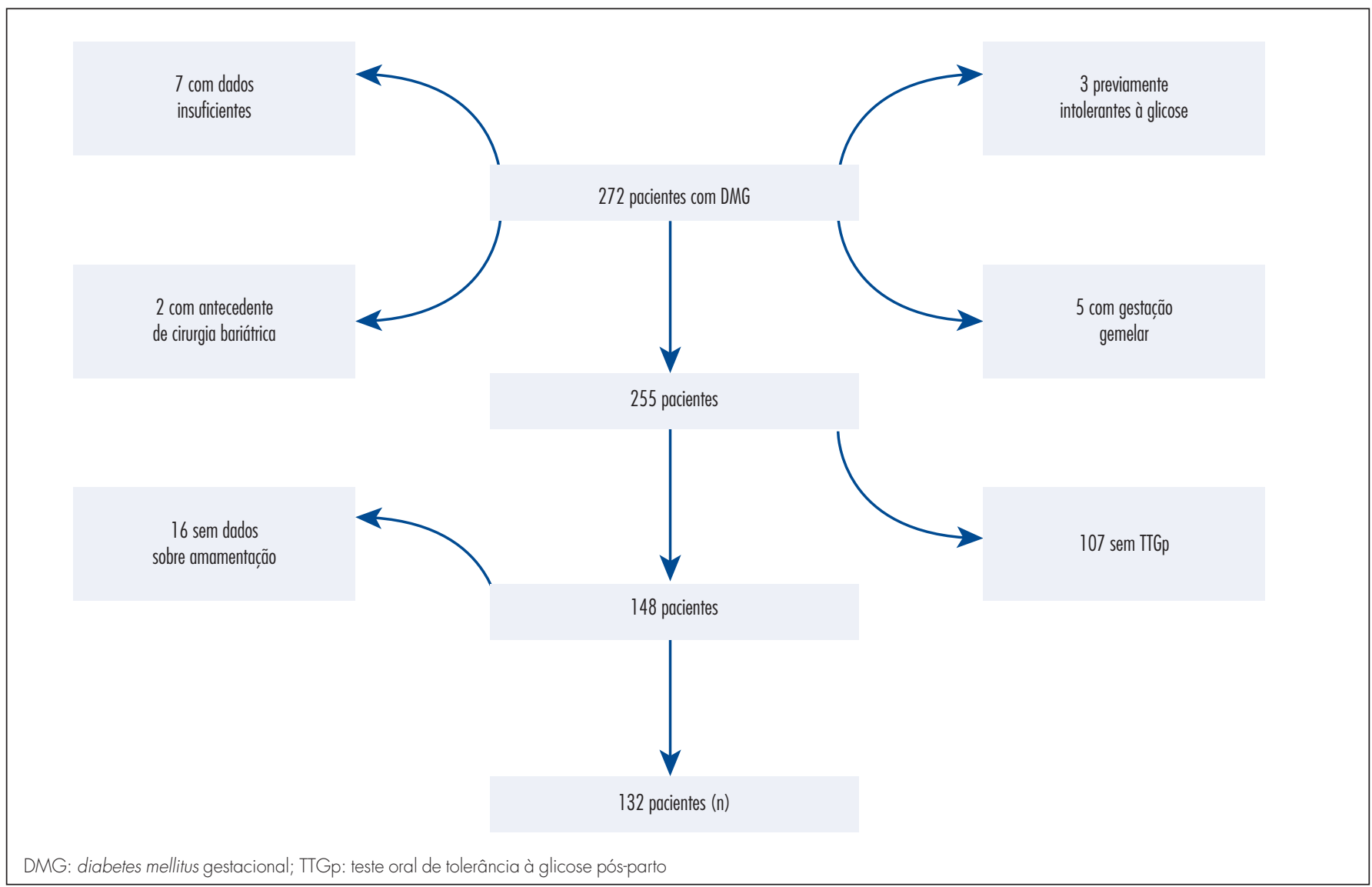

Figura 1. Fluxograma para obtenção final da amostra 
cirurgia bariátrica, 5 com gestações gemelares, 7 sem dados completos no prontuário eletrônico, 107 sem resultados dos valores de TTGp e 16 sem dados sobre amamentação) e as restantes (132 pacientes) foram divididas em dois grupos para avaliação dos dados: as que amamentaram (AS; $\mathrm{n}=114)$ e as que não amamentaram ( $\mathrm{AN} ; \mathrm{n}=18)$.

Não houve diferença entre os grupos estudados quanto à idade, à cor, à paridade, ao antecedente familiar de DM, ao antecedente pessoal de DMG e ao antecedente de feto macrossômico (Tabela 1). A maior parte das pacientes encontra-se acima do peso ideal (sobrepeso/obesidade (88,2\% grupo AN versus $67,9 \%$ grupo AS)) em ambos os grupos, apesar de haver diferença significativa apenas no índice de massa corporal (IMC) pré-gestacional $(\mathrm{p}=0,006)$, menor no grupo que amamentou. $\mathrm{O}$ ganho de peso total também foi diferente entre os grupos, sendo maior no grupo AS $(9,8 \pm 11,2$ versus $5,3 \pm 3,4$ no grupo AN; $\mathrm{p}=0,02)$. Apesar disso, o ganho de peso em ambos os grupos está dentro dos parâmetros recomendados pelo Institute of Medicine ${ }^{19}$.

Em relação à análise do perfil laboratorial e de diagnóstico de DMG, no grupo AN, o diagnóstico foi feito majoritariamente pela GJ $(55,6 \%)$, enquanto no grupo AS a maior parte do diagnóstico foi feita pelo TTG $(60,5 \%)$. Entretanto, não houve diferença significativa entre os grupos quanto ao tipo de exame que estabeleceu

Tabela 1. Características clínicas das pacientes, segundo a divisão por grupos

\begin{tabular}{|c|c|c|c|}
\hline \multirow[b]{2}{*}{ Variável } & \multicolumn{2}{|c|}{ Amamentação } & \multirow[b]{2}{*}{ Valor $p$} \\
\hline & $\begin{array}{c}\text { Não }(n=18) \\
\text { Média } \pm D P / \\
n(\%)\end{array}$ & $\begin{array}{c}\text { Sim }(n=118) \\
\text { Média } \pm \text { DP / } \\
n(\%)\end{array}$ & \\
\hline Idade (anos) & $35 \pm 5$ & $33 \pm 6$ & $0,30^{\circ}$ \\
\hline $\begin{array}{l}\text { Cor } \\
\text { Branca } \\
\text { Não branca }\end{array}$ & $\begin{array}{c}14(77,8) \\
4(22,2)\end{array}$ & $\begin{array}{l}68(59,6) \\
46(40,4)\end{array}$ & 0,19 \\
\hline $\begin{array}{l}\text { Paridade } \\
\text { Primigesta } \\
\text { Secundigesta } \\
\geq 3 \text { gestações }\end{array}$ & $\begin{array}{l}3(16,7) \\
7(38,9) \\
8(44,4)\end{array}$ & $\begin{array}{c}34(29,8) \\
31(27,2) \\
49(43)\end{array}$ & $\begin{array}{l}0,28 c \\
0,4 c \\
1,0 c\end{array}$ \\
\hline AF DM & $12(70,6)$ & $62(54,4)$ & 0,2 \\
\hline AP DMG & $3(17,6)$ & $9(8,0)$ & $0,1^{d}$ \\
\hline Antecedente de macrossomia & $1(5,9)$ & $17(15,3)$ & $0,4^{d}$ \\
\hline $\begin{array}{l}\text { Peso (kg) } \\
\text { Pré-gestacional } \\
\text { Última consulta } \\
\text { Ganho de peso total }\end{array}$ & $\begin{array}{c}76,97 \pm 15,8 \\
82,34 \pm 16,3 \\
5,37 \pm 3,4\end{array}$ & $\begin{array}{c}71,7 \pm 15,8 \\
82,26 \pm 15,3 \\
9,88 \pm 11,2\end{array}$ & $\begin{array}{l}0,2^{0} \\
0,9^{0} \\
0,024^{b}\end{array}$ \\
\hline $\begin{array}{l}\text { IMC } \\
\text { Pré-gestacional } \\
3^{\circ} \text { trimestre } \\
\text { Baixo peso/normal } \\
\text { Sobrepeso/obesidade }\end{array}$ & $\begin{array}{c}31,7 \pm 5,3 \\
32,8 \pm 5,9 \\
2(11,8) \\
15(88,2)\end{array}$ & $\begin{array}{c}27,9 \pm 5,2 \\
31,77 \pm 5,09 \\
35(32,1) \\
74(67,9)\end{array}$ & $\begin{array}{l}0,006^{0} \\
0,4^{0} \\
0,1^{d}\end{array}$ \\
\hline
\end{tabular}

DP: desvio padrão; AF DM: antecedente familiar de diabetes mellitus; AP DMG: antecedente pessoal de diabetes mellitus gestacional; SOP: síndrome dos ovários policísticos; IMC: índice de massa corporal; a: teste t de Student; ${ }^{b}$ : teste U de Mann-Whitney; c: teste do $\chi^{2}$; d: teste exato de Fisher. o diagnóstico (Tabela 2). Em ambos os grupos houve predomínio de tratamento do DMG com dieta $[12$ $(66,7 \%)$ no grupo AN versus 87 (76,3\%) no grupo AS], porém não há diferença significante quanto ao tipo de tratamento. Entre as pacientes que utilizaram insulina para o tratamento, houve diferença significativa quanto à idade gestacional de introdução da medicação, sendo mais precoce no grupo que não amamentou $(23,2 \pm 4,3$ no grupo AN versus 28,8 $\pm 6,1$ no AS; $\mathrm{p}=0,04)$.

No que diz respeito ao seguimento pós-parto (Tabela 2), ao analisar o TTGp, verificamos diferença significante nos valores de GJ entre os grupos, sendo que o grupo que não amamentou apresentou valor médio superior ao grupo que amamentou $(91,3 \pm 8,7$ no grupo $A N$ versus $86,5 \pm 9,3$ no AS; $p=0,01)$. Quando foi avaliada a influência da amamentação na classificação do TTGp, observou-se também diferença significante entre os grupos $(p=0,02)$, com a amamentação agindo como fator protetor para o desenvolvimento de resultados alterados no TTGp $(\mathrm{OR}=0,27$ IC95\% 0,09-0,8). Ao aplicar o modelo de regressão logística com as variáveis ganho de peso total, IMC pré-gestacional e amamentação para determinar quais

Tabela 2. Características quanto ao teste diagnóstico, perfil laboratorial, tratamento e seguimento puerperal das pacientes, segundo a divisão por grupos

\begin{tabular}{|c|c|c|c|}
\hline \multirow[b]{2}{*}{ Variável } & \multicolumn{2}{|c|}{ Amamentação } & \multirow[b]{2}{*}{ Valor $p$} \\
\hline & $\begin{array}{c}\text { Não }(n=18) \\
\text { Média } \pm D P / \\
n(\%)\end{array}$ & $\begin{array}{c}\text { Sim }(n=118) \\
\text { Média } \pm D P / \\
n(\%)\end{array}$ & \\
\hline $\begin{array}{l}\text { Teste diagnóstico } \\
\text { GJ } \\
\text { TTG }\end{array}$ & $\begin{array}{l}10(55,6) \\
8(44,4)\end{array}$ & $\begin{array}{l}43(37,7) \\
71(62,3)\end{array}$ & $0,1^{c}$ \\
\hline GJ $1^{\circ}$ trimestre $(\mathrm{mg} / \mathrm{dL})$ & $93,6 \pm 10,7$ & $88,1 \pm 10,9$ & $0,05^{\circ}$ \\
\hline $\begin{array}{l}\text { Resultados do TG (mg/dL) } \\
\text { GJ } \\
1^{a} \text { hora } \\
2^{a} \text { hora }\end{array}$ & $\begin{array}{c}88,1 \pm 8,5 \\
160,1 \pm 20,4 \\
146,1 \pm 27,9\end{array}$ & $\begin{array}{c}90 \pm 11,5 \\
166,3 \pm 29,1 \\
155,6 \pm 31,1\end{array}$ & $\begin{array}{l}0,6^{0} \\
0,5^{0} \\
0,3^{0}\end{array}$ \\
\hline $\begin{array}{l}\text { Tratamento } \\
\text { Dieta } \\
\text { Insulina }\end{array}$ & $\begin{array}{l}12(66,7) \\
6(33,3)\end{array}$ & $\begin{array}{l}87(76,3) \\
26(22,8)\end{array}$ & $0,4^{d}$ \\
\hline $\begin{array}{l}\text { IG introdução insulina } \\
\text { (semanas) }\end{array}$ & $23,21 \pm 4,33$ & $28,84 \pm 6,17$ & $0,04^{a}$ \\
\hline $\begin{array}{l}\text { Dose final total insulina } \\
\text { (UI) }\end{array}$ & $51,3 \pm 9,8$ & $40,7 \pm 14$ & $0,08^{a}$ \\
\hline HbAlc (\%) & $5,3 \pm 0,5$ & $5,2 \pm 0,4$ & $0,6^{0}$ \\
\hline Frutosamina (mMol/mL) & $201,8 \pm 18,5$ & $196 \pm 19,6$ & $0,2^{0}$ \\
\hline$\Delta t T T G p$ (dias) & $40,9 \pm 16,5$ & $46,7 \pm 24,6$ & $0,4^{b}$ \\
\hline $\begin{array}{l}\text { Resultados do } \Pi \mathrm{Gp}(\mathrm{mg} / \mathrm{dL}) \\
\text { GJ } \\
2^{\text {a }} \text { hora }\end{array}$ & $\begin{array}{c}91,3 \pm 8,7 \\
114,7 \pm 35,5\end{array}$ & $\begin{array}{c}86,5 \pm 9,3 \\
107,7 \pm 33,9\end{array}$ & $\begin{array}{l}0,017^{b} \\
0,5^{b}\end{array}$ \\
\hline $\begin{array}{l}\text { Classificação do TTGp } \\
\text { Normal } \\
\text { Alterado }\end{array}$ & $\begin{array}{l}11(61,1) \\
7(38,9) \\
\end{array}$ & $\begin{array}{l}97(85,1) \\
17(14,9) \\
\end{array}$ & $0,02^{d}$ \\
\hline
\end{tabular}

DP: desvio padrão; GJ: glicemia de jejum; TTG: teste de tolerância à glicose; IG: idade gestacional; $\mathrm{HbAlc}$ : hemoglobina glicada; $\Delta \uparrow T G p$ : intervalo de tempo entre o parto o teste de tolerância à glicose puerperal; a: teste $t$ de Student; b. teste $U$ de Mann-Whitney; c : teste exato de Fisher; ${ }^{d}$ : teste do $\chi^{2}$. 
estavam independentemente relacionadas ao desfecho, a amamentação mostrou-se ser variável independente relacionada à proteção $(\mathrm{p}=0,04)$, enquanto as demais não.

\section{Discussão}

A presente coorte retrospectiva encontrou relação importante entre a amamentação e a diminuição do risco de desenvolver intolerância à glicose e/ou diabetes do tipo 2. Quem amamentou teve chance $73 \%$ menor de desenvolver alterações nos resultados do TTGp. A média da GJ puerperal do grupo que amamentou foi menor, quando comparada às pacientes que não amamentaram, enquanto a glicemia após $2 \mathrm{~h}$ de sobrecarga de glicose foi maior no grupo que não amamentou. Os dados do presente estudo são semelhantes aos encontrados em trabalho que analisou 809 mulheres e encontrou diferença de 5 e $10 \mathrm{mg} / \mathrm{dL}$ a menos na GJ e $2 \mathrm{~h}$ pós-sobrecarga de glicose, respectivamente, em pacientes que amamentaram em relação às que não amamentaram, sem quantificar nem qualificar a amamentação, provando que a amamentação é um fator protetor, independentemente da duração e da qualidade ${ }^{20}$.

Em outro estudo, que considerou o tipo de amamentação, os grupos foram divididos em aleitamento materno exclusivo, aleitamento materno predominante, aleitamento misto ou dados inconsistentes e predomínio de fórmula láctea ou sem aleitamento materno ${ }^{18}$. Concluiu-se que a amamentação é um fator protetor e que, de forma gradativa, quanto menos se amamenta, maior é o risco de desenvolver intolerância à glicose e diabetes do tipo 2 . No presente estudo, as pacientes foram divididas apenas em dois grupos, segundo a informação de terem ou não amamentado, uma vez que o número de participantes foi pequeno e, por ser uma análise retrospectiva de dados, as informações referentes à amamentação ser mista ou exclusiva não eram fidedignas. Dessa forma, optou-se por considerar apenas se a paciente amamentou ou não.

O IMC elevado é um fator de risco isolado para o desenvolvimento de diabetes. Foi observada diferença significativa entre os grupos AS e AN quanto ao IMC pré-gestacional e constatado que as pacientes que amamentaram apresentavam menor IMC em relação àquelas que não amamentaram. Esse dado poderia ser considerado fator de confusão para o desfecho de desenvolver ou não intolerância à glicose e DM2; entretanto, ao realizar a regressão logística, foi constatado que a amamentação é um fator protetor isolado.

Dentre os fatores que estão associados ao abandono do aleitamento precocemente e a não amamentação, encontram-se a obesidade e o sobrepeso. Um estudo concluiu que tanto a obesidade quanto o ganho inadequado de peso durante a gestação têm efeito negativo na prática da amamentação e que essas mulheres necessitam de suporte extra para amamentarem ${ }^{21}$. Os mecanismos que explicam essa relação ainda não estão completamente estabelecidos, porém sabe-se que a obesidade pode produzir alterações no eixo hipotálamo-hipófise-ovariano e no metabolismo lipídico, ocasionando a diminuição da produção láctea ${ }^{22,23}$. Também estão relacionados a essa dificuldade os fatores psicossociais e anatômicos, visto que mamas muito volumosas dificultam a técnica de pega e posição para amamentação ${ }^{24,25}$. Um dos fatores que podem estar relacionados ao fato de as pacientes do presente estudo não terem amamentado, portanto, é o IMC pré-gestacional elevado.

Em meta-análise publicada em 2013, após análise de estudos de coorte prospectivas, constatou-se que a maior duração da amamentação de forma exclusiva, quando comparada a não amamentação, apresentou uma redução de $32 \%$ no risco relativo de desenvolver DM2 em pacientes que tiveram $\mathrm{DMG}^{14}$. Em análise de dose-resposta linear, observou-se diminuição significativa de $9 \%$ em tal risco relativo a cada 12 meses de amamentação. Além disso, verificou-se que a amamentação é fator protetor isolado, independentemente de outros importantes fatores, como peso, IMC, tabagismo, alcoolismo, prática de exercícios físicos, classe socioeconômica, história familiar de diabetes e paridade. O presente estudo, embora seja uma coorte retrospectiva com pequeno número de participantes, também identificou a amamentação como fator protetor ao desenvolvimento de intolerância à glicose.

Os resultados do presente estudo são semelhantes aos encontrados em outro trabalho, no que diz respeito aos fatores de risco para desenvolvimento de DM2. As pacientes que tiveram DMG foram acompanhadas durante 19 anos após o parto para avaliar quais fatores influenciavam o surgimento de DM2. Ao final do estudo, verificou-se que o tipo de tratamento recebido na gestação (dieta ou insulina), o IMC e a presença ou ausência de autoanticorpos eram fatores de risco para o desenvolvimento de DM2. Além disso, mulheres que amamentaram tiveram DM2 12,3 anos após o parto, enquanto as que não amamentaram desenvolveram esse desfecho em 2,3 anos. Concluiu-se, também, que a amamentação é melhor fator protetor quando se dá por mais de 3 meses e que reduz em mais de $40 \%$ o risco em longo prazo de desenvolver DM $2^{11}$. Assim como no presente estudo, os autores também não classificaram a amamentação como mista ou exclusiva.

Algumas limitações podem ser observadas no presente estudo. A coleta de dados se deu ao longo de um ano e apenas $54 \%$ das pacientes retornam para realizar TTGp, o que tornou o tamanho amostral pequeno para adequada análise estatística. Como pouco mais da metade das pacientes não retornou, é possível refletir que é necessária uma melhor orientação, durante todo o período de 
pré-natal e internação para o parto, sobre a importância do seguimento puerperal, visando a conscientização sobre a presença de tal distúrbio metabólico em algum momento de suas vidas e as formas de evitar esse desfecho, como orientações de dieta balanceada e fracionada, manutenção de IMC dentro dos padrões da normalidade e prática regular de atividade física.

Embora a informação sobre amamentação não sofra o efeito Hawthorne, visto que a coleta de dados foi retrospectiva e as participantes não sabiam sobre a existência do estudo, há a possibilidade de parte das pacientes não ter informado corretamente sobre esse dado.

A amamentação é uma intervenção efetiva de baixo custo, de fácil acesso a todas as pacientes no puerpério e que deve ser estimulada, pois, além de fornecer múltiplos benefícios ao recém-nascido, diminui o risco de mães que tiveram diagnóstico de DMG desenvolverem intolerância à glicose e DM2.

\section{Referências}

1. Zugaib M. Diabetes mellitus. In: Zugaib $M$, editor. Zugaib Obstetrícia. Barueri: Manole; 2012. p. 892-905.

2. HAPO Study Cooperative Research Group. Hyperglycemia and adverse pregnancy outcomes. N Engl J Med. 2008;358(19): 1991-2002.

3. International Association of Diabetes and Pregnancy Study Groups Consensus Panel.International Association of Diabetes and Pregnancy Study Groups recommendations on the diagnosis and classification of hyperglycemia in pregnancy. Diabetes Care. 2010;33(3):676-82.

4. American Diabetes Association. Diagnosis and classification of diabetes mellitus. Diabetes Care.2014;37(Suppl1):S81-90.

5. Massucatti LA, Pereira RA, Maioli TU. Prevalência de diabetes gestacional em Unidades de Saúde Básica. REAS. 2012;1 (1):70-9.

6. American Diabetes Association. Classification and diagnosis of diabetes. Diabetes Care. 2015;38(Suppl):S8-S16.

7. Bellamy L, Casa JP, Hingorani AD, Williams D. Type 2 diabetes mellitus after gestational diabetes: a systematic review and metaanalysis. Lancet. 2009;373(9677): 1773-9.

8. Francisco RPV, Trindade TC. Diabetes gestacional. In: Zugaib M, Bittar RE, editores. Protocolos assistenciais clínica obstétrica FMUSP. 4a ed. São Paulo: Atheneu; 2011 . p. 243-51.

9. Gunderson EP, Crites Y, Chiang V, Walton D, Azevedo RA, Foz $G$, et al. Influence of breastfeeding during the postpartum oral glucose tolerance test on plasma glucose and insulin. Obstet Gynecol. 2012;120(1):136-43.

10. Colatrella A, Framarino M, Toscano V, Bongiovanni M, Festa $C$, Mattei $L$, et al. Continuous glucose monitoring during breastfeeding in women with recent gestational diabetes mellitus. Diabetes Technol Ther. 2012;14(7):576-82.

11. Ziegler AG, Wallner $M$, Kaiser I, Rossbaver $M$, Harsunen $M H$, Lachmann $L$, et al. Long-term protective effect of lactation in the development of type 2 diabetes in women with recent gestational diabetes mellitus. Diabetes. 2012;61(12):3167-71.

12. O'Reilly MW, Avalos G, Dennedy MC, O'Sullivan EP, Dunne F. Atlantic DIP: high prevalence of abnormal glucose tolerance post partum is reduced by breast-feeding in women with prior gestational diabetes mellitus. Eur J Endocrinol. $2011 ; 165(6): 953-9$.
13. Chouinard-Castonguay S, Weisnagel SJ, Tchernof A, Robitaille J. Relationship between lactation duration and insulin and glucose response among women with prior gestational diabetes. Eur J Endocrinol. 2013;168(4):515-23.

14. Aune D, Norat T, Romundstad P, Vatten L. Breastfeeding and the maternal risk of type 2 diabetes: a systematic review and doseresponse meta-analysis of cohort studies. Nutr Metab Cardiovasc Dis. 2014;24(2): 107-15.

15. Nelson AL, Le MH, Musherraf Z, Vanberckelaer A. Intermediateterm tolerance in women with a history of gestational diabetes: natural history and potential associations with breastfeeding and contraception. Am J Obstet Gynecol. 2008;198(6):699. e 1-7.

16. Much D, Beyerlein A, Roßbauer M, Hummel S, Ziegler AG.Beneficial effects of breastfeeding in women with gestational diabetes mellitus. Mol Metab. 2014;3(3):284-92.

17. Gunderson EP. Impact of breastfeeding on maternal metabolism: implications for women with gestational diabetes. Curr Diab Rep. $2014 ; 14(2): 460$.

18. Gunderson EP, Hedderson MM, Chiang V, Crites Y, Walton D, Azevedo RA, et al. Lactation intensity and postpartum maternal glucose tolerance and insulin resistance in women with recent GDM: the SWIFT cohort. Diabetes Care. 2012;35(1):50-6.

19. Rasmussen KM, Yaktine A; Institute of Medicine. Weight gain during pregnancy: reexamining the guidelines. Washington (DC): National Academies Press; 2009.

20. Kjos SL, Henry O, Lee RM, Buchanan TA, Mishell DR Jr. The effect of lactation on glucose and lipid metabolism in women with recent gestational diabetes. Obstet Gynecol. 1993;82(3):451-5.

21. Li R, Jewell S, Grummer-Strawn L. Maternal obesity and breastfeeding practices. Am J Clin Nutr. 2003;77(4):931-6.

22. Bray GA. Obesity and reproduction. Hum Reprod. 1997; 12Suppl 1:26-32.

23. Rasmussen KM, Hilson JA, Kjolhede CL. Obesity may impair lactogenesis II. J Nutr. 2001;131(11):3009S-11S.

24. Chapman DJ,Pérez-Escamilla R. Identification of risk factors for delayed onset of lactation. J Am Diet Assoc. 1999;99(4):450-4.

25. Morrison MK, Collins CE, Lowe JM, Giglia RC. Factors associated with early cessation of breastfeeding in women with gestational diabetes mellitus. Women Birth. 2015;28(2):143-7. 